\title{
BMJ Open Clinical preventive guidelines for school-aged children and adolescents in primary care: a protocol for a systematic review
}

\author{
Magdalini Patseadou (D) , ${ }^{1,2}$ Eva Pfarrwaller, ${ }^{1}$ Dagmar M Haller (D) ${ }^{1,3}$
}

To cite: Patseadou M,

Pfarrwaller E, Haller DM. Clinical preventive guidelines for schoolaged children and adolescents in primary care: a protocol for a systematic review. BMJ Open 2020;10:e037396. doi:10.1136/ bmjopen-2020-037396

- Prepublication history and supplemental material for this paper is available online. To view these files, please visit the journal online (http://dx.doi. org/10.1136/bmjopen-2020037396).

Received 31 January 2020 Revised 14 November 2020 Accepted 27 November 2020

Check for updates

(c) Author(s) (or their employer(s)) 2020. Re-use permitted under CC BY-NC. No commercial re-use. See rights and permissions. Published by BMJ.

${ }^{1}$ Primary Care Unit, Faculty of Medicine, University of Geneva, Geneva, Switzerland

${ }^{2}$ Department of Woman, Child and Adolescent Health, Geneva University Hospitals, Geneva, Switzerland

${ }^{3}$ Department of Primary Care Medicine, Geneva University Hospitals, Geneva, Switzerland

Correspondence to

Dr Magdalini Patseadou;

magdalini.patseadou@unige.ch

\section{ABSTRACT}

Introduction Guidelines for clinical preventive services targeting school-aged children and adolescents in primary care are limited, often inconsistent and difficult to apply in clinical contexts. This publication describes the protocol concerning a comprehensive systematic review that primarily aims to collect and synthesise available guidelines for prevention in primary care focused on school-aged children living in high-income regions. A second objective is to assess the quality of identified documents.

Methods and analysis We will search for reports providing clinical practice guidelines or consensus or expert opinion on preventive actions in paediatric primary care. We will use the WHO definition of prevention. We will focus on children aged 6-18 years living in the European region, the USA, Canada and Australia. We will search PubMed, Embase, Web of Science and Cochrane Library and guidelines-specific databases from 1 January 2010. We will also explore the grey literature using web search engines (Google and Google Scholar). We will finally obtain unpublished information through personal contact with national paediatric societies. We will summarise all identified documents as well as their potential methodological bias. We will further use the Appraisal of Guidelines Research and Evaluation Instrument, version II tool to critically appraise their quality.

Ethics and dissemination Our findings will contribute to the identification of clinical preventive guidelines for which implementation in routine paediatric primary care should be considered. We intend to disseminate our results through publication in peer-reviewed journals and conference proceedings.

PROSPERO registration number CRD42020163184.

\section{INTRODUCTION}

Primary care physicians, in particular paediatricians, recognise prevention as a central part of their mission towards patients. Clinical practice guidelines (CPGs) are valuable tools that can assist paediatricians in their preventive activities and improve the quality of care for young patients. ${ }^{1}$ Guidance focused on the prevention of a set of specific diseases and conditions in children is well documented ${ }^{2-6}$; however, it appears
Strengths and limitations of this study

- The present study will be the first systematic review to identify and assess the quality of available guidance on clinical preventive services in school-aged children and adolescents in European high-income countries, the USA, Canada and Australia.

- The extensive search strategy covering both indexed and grey literature as well as personal contact with different study groups is an important strength.

- The input of a national as well as an international advisory group will limit the risk of missing important documents.

- The application of the Appraisal of Guidelines Research and Evaluation Instrument, version II tool, which has established validity and reliability, will enable us to appraise the quality of guidelines.

- A limitation will be the exclusion of documents regarding high-income countries other than the European region, the USA, Canada or Australia.

that guidance addressing the broader concept of preventive primary healthcare throughout childhood and adolescence is rather limited, particularly outside the USA and Canada. ${ }^{7}$ In fact, availability of high-quality studies conducted in paediatric primary care settings remains insufficient because of the general lack of research interest in the area of prevention as well as the multiple barriers to conducting research in children populations. ${ }^{89}$

Globally, there is a plethora of guidelines regarding regular health maintenance visits (well-child visits) for children up to the age of 5. During these visits, basic immunisations as well as monitoring of growth and development are ensured. Yet, provision of clinical preventive care in the postschool entry period is particularly problematic. ${ }^{10}$ Due to the absence of routine scheduled immunisations, older children and adolescents often consult their doctors in case of acute illness or injury, therefore, potentially benefitting only from opportunistic preventive counselling. ${ }^{11}{ }^{12}$ Besides, in certain countries, 
preventive medicine appointments and related costs are not reimbursed by health insurers (the case in Switzerland), thus leading to unavoidable inequalities in access to preventive healthcare. $^{13-15}$

Available guidelines for primary care prevention in paediatric populations usually come in the form of long checklists describing a variety of detailed assessments to be done in each age group. However, these lists are difficult to integrate into the busy routine of primary care practice, a fact that contributes to their underuse by clinicians. ${ }^{16}$ The development of brief essential recommendations reduces time lost due to unnecessary activities and, therefore, reduces unnecessary costs.

CPGs' trustworthiness is an important prerequisite to promoting their use. ${ }^{17}$ Potential inconsistencies observed between different study groups that develop CPGs are mainly due to different methodological approaches applied. The development of low-quality guidelines has significant implications when implementing them in clinical practice in terms of both absence of health benefits to patients and potential risk of harm as well as wasted resources. ${ }^{19}{ }^{20}$ On the other hand, it is well observed that certain countries proceed to full endorsement of evidence-based guidance that has been previously published by specific trustworthy groups from other countries. However, local epidemiology and practice contexts, medical costs, as well as ethnical and social differences, play a significant role in defining each country's specific healthcare needs and should be taken into consideration when establishing national CPGs.

In view of this, our aim is to review all available clinical preventive guidelines regarding school-aged children in high-income countries and identify key effective preventive services for which implementation in routine paediatric primary care should be considered.

\section{Objective}

Our main objective is to collect, summarise and assess the quality of available guidelines for prevention in primary care targeting school-aged children and adolescents living in European high-income regions, the USA, Canada and Australia. Our secondary objective is to create a list of preventive services with evidence supporting their routine use in primary care.

\section{METHODS AND ANALYSIS}

This protocol is prepared based on the Preferred Reporting Items for Systematic Reviews and Meta-Analyses (PRISMA) for Protocols ${ }^{21}$ (online supplemental file 1).

\section{Patient and public involvement}

No patient involved.

\section{Eligibility criteria}

We will include all available guidance for prevention in school-aged children in European high-income countries, as well as the USA, Canada and Australia.
We define school-aged children as those between 6 and 18 years. We will also consider reports concerning recommendations for the full range of the paediatric population, namely from birth to 18 years, and we will screen them by hand in order to identify specific guidance for children and adolescents aged 6-18 years.

For identifying high-income countries, we will use the WHO classification of countries by income level, which is based on the World Bank list. ${ }^{22}$ We will focus on the European region, since the needs in disease prevention and health promotion in Switzerland are quite similar to those met in the rest of the high-income European countries. We will further include the USA, Canada and Australia because historically these countries have led the way in following rigorous standards when developing clinical guidelines. We choose not to include other highincome countries in order to focus on information that will be specifically relevant to the European context, and also because access to data from these other countries is likely to be limited by language/writing barriers.

We will include guidance for preventive services that result in the maintenance and promotion of health in the general paediatric and adolescent population. As such, we refer to every action that aims at avoiding or detecting the manifestation of disease or injury, in line with the WHO definition for primary and secondary prevention. ${ }^{23}$ This may include vaccinations, provision of information and education on behavioural health risks and measures to reduce these risks, actions to improve health through changing the impact of certain social and economic determinants, nutritional and food supplementation, dental hygiene education and oral health services, as well as evidence-based screening programmes for early detection of illness.

We will focus on preventive activities implemented in primary care settings. Primary care is a whole-to-society approach to health and well-being, characterised by accessible and comprehensive healthcare, centred on the needs of individuals and communities. It addresses the broader determinants of health and focuses on the interrelated aspects of physical, mental and social health and well-being. ${ }^{23}$

We will initially search for CPGs. We will use the CPGs definition proposed by the Institute of Medicine according to which, every statement that includes recommendations intended to optimise patient care and that is informed by a systematic review of evidence and an assessment of the benefits and harms of alternative care options, is considered a CPG. ${ }^{1}$ We will further search for consensus statements, recommendations and position papers based on expert opinions, as we expect that the number of available CPGs will be limited.

We will examine all documents published in languages understood by the members of our research team, namely English, French, German, Italian, Spanish, Greek, Portuguese, Swedish and Dutch. As we assume that there is a high probability of identifying a large number of publications and some of them will be updates of documents 
issued by the same group, we will limit our search to the last 10 years, and we will update it before publishing our final findings.

We will exclude older versions of identified documents if previously issued by the same organisation.

\section{Search strategy}

1. We will search for published guidelines concerning paediatric preventive care in the following scientific databases: PubMed, Embase, Web of Science and Cochrane Library. We will use combinations of the following free-text words and medical subject headings (MeSH terms) as well as their synonyms : 'guideline', 'recommendation', 'statement', 'consensus,' 'prevention', 'primary care', 'child' and 'adolescent' (for details on the search methodology concerning PubMed, see online supplemental file 2). We will additionally search for relevant links and citations through hand screening of reference lists of all documents included in full-text screening.

2. We will also search databases specific to guidelines: the Guidelines International Network, the Guidelines and Audit Implementation Network, the Institute for Clinical Systems Improvement, the Turning Research into Practice, the WHO, the Epistemonikos, the National Institute for Health and Care Excellence, the National Health Service Evidence, the Canadian Medical Association Infobase, the Scottish Intercollegiate Guidelines Network and the Australian CPGs.

3. We will further expand our search outside of scientific databases. For that reason, we will search grey literature using Web engines, mainly Google and Google Scholar. We will use some of the same free-text search words, namely: 'guidelines', 'recommendations', 'statement', 'consensus', 'prevention', 'preventive', 'children', 'adolescents', 'primary care' (for details on the search strategy on Google and Google Scholar, see online supplemental file 3). We will be limited to the form of pdf files and we will screen the first 200 records for each search string. Besides, we will systematically search publications in the websites of all relevant national paediatric societies and associations.

4. We also consider obtaining unpublished or published information in languages other than those already specified for the study purposes, through personal contact with representatives from relevant national paediatric organisations. If a response is not received after three contact attempts, we will exclude the relevant society from the review.

\section{Guidelines selection process}

We will import retrieved references from each database into EndNote X9 citation manager software (Clarivate Analytics, Philadelphia, USA). This will allow for systematic storage as well as the ability to remove duplicate documents. Then, we will import references into Covidence (Veritas Health Innovation, Melbourne Australia), a web-based standardised systematic review platform, to facilitate the screening process. The primary reviewer (MP) will select articles by reading the title and the abstract after having ensured consistency with the second reviewer (EP) who will independently screen the first 200 references. Finally, the two reviewers will blindly undertake the full-text screening. Potential disagreements will be resolved through discussion with the third reviewer (DH).

The documents selection and review process will be demonstrated on a PRISMA flow chart. Reasons for exclusion will be reported in the full-text screening step.

\section{Data extraction and management}

The two reviewers will independently extract data from each eligible document to Covidence data extraction form. The following data will be extracted: first author, year of publication, country, title, issuing organisation, funding body, target population, health topic (we anticipate the following key areas: nutrition, physical activity, obesity, hypertension, oral health, vaccines and immunisation, violence \& injuries, substance use, sexual health, mental health) content of recommendation, method used to formulate guidance (systematic review, consensus, position paper, not mentioned, other), methodological quality (method of grading the evidence), if any, with relevant strength of recommendation (high, low), if any. We will contact the corresponding authors of the identified documents by email for questions about any available information that is not included in the published documents but needed for the analysis.

\section{Critical appraisal of quality}

We will assess eligible guidelines using the Appraisal of Guidelines Research and Evaluation Instrument, V.II (AGREE II), an internationally well-validated tool for the evaluation of the quality of evidence and the development process of clinical guidelines. ${ }^{24}$ This instrument consists of 23 items grouped in six domains: scope and purpose, stakeholder involvement, rigour of development, clarity and presentation, applicability, and editorial independence. Each item is scored on a Likert scale of seven points from strongly disagree to strongly agree (1-7, respectively).

The two reviewers will perform the appraisal of guidance after having completed the online AGREE II training. Inter-rater reliability will be assessed by the intraclass correlation coefficient (ICC) which will be calculated for each domain of the AGREE II. An ICC $\geq 0.7$ will be considered acceptable. ${ }^{25}$ A standardised quality scaled score will be calculated for each of the six AGREE II domains as follows:

Scaled Domain Score $=($ Obtained score-Minimum possible score)/(Maximum possible score-Minimum possible score) $\mathrm{x} 100$

For each domain: The maximum score will be derived by multiplying the number of items included in this domain by two (since two reviewers), multiplied by 7 ('strongly agree'). Accordingly, the minimum score will 
be derived by multiplying the number of items in this domain by 2 , multiplied by 1 ('strongly disagree'). The 'obtained score' will be calculated as the sum of scores given by the two reviewers for all the items included in this domain.

\section{Results synthesis}

We expect that in the present systematic literature review, data will not be appropriate to be used in meta-analysis. Instead, we will present our results narratively. We will tabulate descriptive data of all eligible documents. If possible, we will group recommendations by topic (health condition), location and age group (we anticipate five age subgroups: $6-8,9-12,13-15$ and $16-18$ years). We will comment on the potential methodological bias. We will further demonstrate the scores of AGREE evaluation on a table format. We will finally discuss on the recommendations to be considered for implementation in routine primary care.

\section{Advisory Committee}

For the purpose of this study, we have created an advisory committee that includes national and international experts with great experience in both primary care and the development of preventive guidelines (see online supplemental file 4 for complete list). Their contribution will consist of commenting the list of guidelines identified by the research team for further analysis and suggesting additional sources of information potentially missed. They will further be asked to assess the summary of findings of the review and comment on the synthesis of evidence-based recommendations.

\section{ETHICS AND DISSEMINATION}

Ethical approval is not required for this systematic review because all data included in the review have been either published or are publicly available.

The purpose of this review is to identify rigorous recommendations for clinical preventive services in school-aged children. In a second step, we will adapt these identified recommendations to the Swiss epidemiological and practice context to create a list of clinically relevant and evidence-based practices. Thereafter, we aim to conduct a Delphi consensus study involving community paediatricians and general practitioners throughout Switzerland to define maximum five essential preventive activities to prioritise from the created list for each school-age group (6-8, 9-12, 13-15 and 16-18 years). We hope that our final findings will provide valuable information for Swiss stakeholders and decision-makers in planning better delivery strategies that overall will improve the quality of children's preventive care. In addition, our methodology and findings will provide an entry point for further similar research in other countries.

We consider submitting a manuscript to a peer-reviewed journal to present the results of this review, as well as brief summaries at national and international meetings.
Correction notice This article has been corrected since it was published. Middle initial added in author name Dagmar M Haller.

Acknowledgements We thank the members of our advisory committee for accepting to share their knowledge and expertise throughout the project (see online supplemental file 4 for complete list). We also thank Ms Mafalda Burri, MSc librarian in the University of Geneva, for her valuable assistance in the development of the search strategy.

Contributors MP is the principal investigator of this review, drafted the protocol and will be the primary reviewer. EP commented on the protocol and will be the second reviewer. DH conceived the original idea of the systematic review, will resolve potential conflicts and critically revised the protocol.

Funding This research is partly supported by the Swiss College of Primary Care Medicine in the context of a grant to promote early stage research programmes.

Disclaimer The funding source neither influenced the development of the protocol nor will have an impact on the conduction of the review. Authors decided to submit the present article for publication entirely independently of the funders.

Competing interests All authors have completed the icmje uniform disclosure form at www.icmje.org/coi_disclosure.pdf and declare that the only support for the submitted work was from the funders mentioned in 'funding sources'. The authors have no financial relationships with any organisations that might have an interest in the submitted work in the previous 3 years and no other relationships or activities that could appear to have influenced the submitted work.

Patient consent for publication Not required.

Provenance and peer review Not commissioned; externally peer reviewed.

Supplemental material This content has been supplied by the author(s). It has not been vetted by BMJ Publishing Group Limited (BMJ) and may not have been peer-reviewed. Any opinions or recommendations discussed are solely those of the author(s) and are not endorsed by BMJ. BMJ disclaims all liability and responsibility arising from any reliance placed on the content. Where the content includes any translated material, BMJ does not warrant the accuracy and reliability of the translations (including but not limited to local regulations, clinical guidelines, terminology, drug names and drug dosages), and is not responsible for any error and/or omissions arising from translation and adaptation or otherwise.

Open access This is an open access article distributed in accordance with the Creative Commons Attribution Non Commercial (CC BY-NC 4.0) license, which permits others to distribute, remix, adapt, build upon this work non-commercially, and license their derivative works on different terms, provided the original work is properly cited, appropriate credit is given, any changes made indicated, and the use is non-commercial. See: http://creativecommons.org/licenses/by-nc/4.0/.

\section{ORCID iDs}

Magdalini Patseadou http://orcid.org/0000-0003-3619-4196

Dagmar M Haller http://orcid.org/0000-0003-1781-3318

\section{REFERENCES}

1 Field MJ, Lohr KN. Clinical practice guidelines: directions for a new program, Institute of medicine. Washington, DC: National Academy Press, 1990.

2 Styne DM, Arslanian SA, Connor EL, et al. Pediatric ObesityAssessment, treatment, and prevention: an endocrine Society clinical practice guideline. J Clin Endocrinol Metab 2017;102:709-57.

3 Global Initiative for Asthma. Global strategy for asthma management and prevention, 2018. Available: https://ginasthma.org/wp-content/ uploads/2019/06/GINA-2019-main-report-June-2019-wms.pdf [Accessed 2 Dec 2019].

4 Oskoui M, Pringsheim T, Billinghurst L, et al. Practice guideline update summary: pharmacologic treatment for pediatric migraine prevention: report of the Guideline development, Dissemination, and Implementation Subcommittee of the American Academy of Neurology and the American headache Society. Neurology 2019;93:500-9.

5 de Silva D, Geromi M, Halken S, et al. EAACl food allergy and anaphylaxis guidelines group. primary prevention of food allergy in children and adults: systematic review. Allergy 2014;69:581-9.

6 Munns CF, Shaw N, Kiely M, et al. Global consensus recommendations on prevention and management of nutritional rickets. Horm Res Paediatr 2016;85:83-106.

7 Melnyk BM, Grossman DC, Chou R, et al. US preventive services Task force. USPSTF perspective on evidence-based preventive recommendations for children. Pediatrics 2012;130:e399-407. 
8 Horwitz SM, Kelleher K, Boyce T, et al. Barriers to health care research for children and youth with psychosocial problems. JAMA 2002;288:1508-12.

9 Cohen E, Goldman RD, Ragone A, et al. Child vs adult randomized controlled trials in specialist journals: a citation analysis of trends, 1985-2005. Arch Pediatr Adolesc Med 2010;164:283-8.

10 Moyer VA, Butler M. Gaps in the evidence for well-child care: a challenge to our profession. Pediatrics 2004;114:1511-21.

11 The Royal Australian College of General Practitioners (RACGP). Guidelines for preventive activities in general practice. 9th edn. East Melbourne, Vic: RACGP, 2018.

12 Ma J, Wang Y, Stafford RS. U.S. adolescents receive suboptimal preventive counseling during ambulatory care. Journal of Adolescent Health 2005;36:441.e1-441.e7.

13 Tylee A, Haller DM, Graham T, et al. Youth-friendly primary-care services: how are we doing and what more needs to be done? Lancet 2007;369:1565-73.

14 Kefford $\mathrm{CH}$, Trevena LJ, Willcock SM. Breaking away from the medical model: perceptions of health and health care in suburban Sydney youth. Med J Aust 2005;183:418-21.

15 Smith KV, Dye C. How well is CHIP addressing primary and preventive care needs and access for children? Acad Pediatr 2015;15:S64-70.

16 Yarnall KSH, Pollak KI, Østbye T, et al. Primary care: is there enough time for prevention? Am J Public Health 2003;93:635-41.

17 Grol R, Dalhuijsen J, Thomas S, et al. Attributes of clinical guidelines that influence use of guidelines in general practice: observational study. BMJ 1998;317:858-61.
18 Bodenheimer T. Transforming practice. N Engl J Med 2008;359:2086-9.

19 Graham R, Mancher M, et al, Institute of Medicine (US) Committee on Standards for Developing Trustworthy Clinical Practice Guidelines. Clinical practice guidelines we can trust. Washington (DC): National Academies Press (US), 2011. https://www.ncbi.nlm. nih.gov/books/NBK209539/ 10.17226/13058

20 Ransohoff DF, Pignone M, Sox HC. How to decide whether a clinical practice guideline is trustworthy. JAMA 2013;309:139

21 Shamseer L, Moher D, Clarke M, et al. Preferred reporting items for systematic review and meta-analysis protocols (PRISMA-P) 2015: elaboration and explanation. BMJ 2015;350:g7647.

22 Country and Lending Groups. World bank. Available: https:// datahelpdesk.worldbank. org/knowledgebase/articles/906519-worldbank-country-and-lending-groups [Accessed 8 Dec 2019].

23 Health Promotion and Disease Prevention. World Health organization. Available: http://www.emro.who.int/about-who/public-healthfunctions/health-promotion-disease-prevention.html [Accessed 4 Nov 2019].

24 Brouwers MC, Kho ME, Browman GP, et al. Agree next steps Consortium. agree II: advancing Guideline development, reporting and evaluation in healthcare. CMAJ 2010;182:E839-2.

25 Brouwers MC, Kho ME, Browman GP, et al. Development of the agree II, part 1: performance, usefulness and areas for improvement. CMAJ 2010;182:1045-52. 